\title{
Kernos
}

Revue internationale et pluridisciplinaire de religion grecque antique

15 | 2002

Varia

\section{La construction d'un « calendrier » en Grèce ancienne : temps du rituel et temps du récit}

Sofia Kravaritou

\section{(2) OpenEdition}

Journals

\section{Édition électronique}

URL : http://journals.openedition.org/kernos/1360

DOI : 10.4000/kernos. 1360

ISSN : 2034-7871

\section{Éditeur}

Centre international d'étude de la religion grecque antique

\section{Édition imprimée}

Date de publication : 1 janvier 2002

ISSN : 0776-3824

\section{Référence électronique}

Sofia Kravaritou, «La construction d'un « calendrier » en Grèce ancienne : temps du rituel et temps du récit », Kernos [En ligne], 15 | 2002, mis en ligne le 21 avril 2011, consulté le 01 mai 2019. URL : http:// journals.openedition.org/kernos/1360 ; DOI : 10.4000/kernos.1360 


\section{La construction d'un « calendrier » en Grèce ancienne: temps du rituel et temps du récit}

Malgré l'absence d'un « livre » établissant le rythme de l'ensemble des cultes en Grèce ancienne, nous disposons d'une documentation très riche, encore que fragmentaire. Faute d'une possibilité de reconstruction de l'histoire complète des calendriers grecs à partir de textes d'origines diverses, l'étude des textes poétiques de l'époque « archaïque » a conduit les chercheurs à postuler l'existence d'un «calendrier primitif » pratique, appliqué dans les cités grecques de l'époque « archaïque ». Plus spécifiquement, l'existence d'un programme annuel fondé notamment sur les phénomènes astronomiques et météorologiques dans Les Travaux et les jours d'Hésiode a été considérée comme une preuve du caractère irrationnel de la construction religieuse du temps à cette époque. Pour dénoncer cette fausse image, nous entendons montrer que l'utilisation des textes poétiques dans le cadre d'études concernant la construction du temps religieux en Grèce ancienne doit prendre en considération la distinction importante entre deux temporalités qui se déploient de façon parallèle : le temps du rituel, qui renvoie à une notion cyclique du temps, et le temps du récit poétique qui se manifeste de façon linéaire. Le « calendrier » issu d'une œuvre poétique doit être étudié en relation avec le programme poétique développé par l'auteur. Il nous semble que le «calendrier » façonné par Hésiode ne peut pas être utilisé comme témoignage d'une présence de l'irrationnel dans la religion grecque.

Reprenant l'ensemble de la problématique, on note que l'étude des indices temporels, issus des poèmes homériques, a été utilisée comme argument pour démontrer qu'à l'époque de leur composition, il n'existait pas dans les cités grecques de calendrier pratique avec des noms de mois et des actes de cultes ordonnés. Plus particulièrement, l'absence des noms de mois chez Homère a été considérée par M.P. Nilsson ${ }^{1}$ comme preuve du fait que

* Les réflexions préliminaires sur le texte hésiodique présentées ici doivent beaucoup aux discussions menées avec $M$. Claude Calame, ainsi qu'aux séminaires de littérature grecque et au séminaire postgrade du Centre de Recherches Interdisciplinaires en Analyse des Discours de l'Université de Lausanne. Je remercie vivement M. David Bouvier pour la lecture du texte et pour ses conseils. Mes remerciements vont également à $M$. Lambros Couloubaritsis, pour ses remarques durant le colloque du CIERGA à Rhodes. La traduction du texte ancien en français reproduit la version de P. Mazon (CUF, 1944).

1 Les études fondamentales de M.P. Nilsson font partie d'une série de recherches représentatives des approches comparatives entre diverses cultures qui caractérisent les 
les Grecs de cette époque n'utilisaient pas de noms pour les intervalles temporels. D'après la phrase homérique : "soit au mois qui finit, soit au mois qui commence " ${ }^{2}$, l'historien des religions explique que les Grecs, même s'ils calculaient le temps à l'aide des mois, n'avaient pas de noms pour ces mois, puisqu'Homère ne les connaît pas ${ }^{3}$. Des études postérieures ont comparé ces résultats avec les indices temporels des Travaux et les jours d'Hésiode et, ils ont conclu à une évolution progressive des calendriers pratiques des cités depuis l'époque de la composition des œuvres homériques jusqu'à l'époque d'Hésiode $^{4}$. L'argument principal invoqué pour démontrer cette évolution se fonde d'abord sur une supposition d'après laquelle on ne distingue guère d'indications d'un «calendrier civil » chez Homère, tandis que chez Hésiode une forme plus élaborée est présentée, puisque il fait aussi mention d'un nom de mois en fonction dans les cités grecques de l'époque historique. Deuxièmement, cette argumentation insiste sur le fait que dans Les Travaux et les jours d'Hésiode le système de calcul des jours de la dernière partie du poème est plus développé. Par ailleurs, cette dernière partie a été considérée comme un addendum postérieur ${ }^{5}$. Pour faire face à cette problématique et avant d'exclure certaines parties du poème, il faut d'abord examiner leur articulation dans la structure de l'ensemble en fonction du programme poétique. Par conséquent, avant d'observer le déploiement de deux temporalités différentes, cyclique et linéaire, à l'intérieur du même poème, il faut pouvoir distinguer les différents plans annuels qui y sont décrits.

Dans la partie du poème connue comme «calendrier hésiodique ${ }^{6}$, le narrateur fait dérouler en premier lieu ${ }^{7}$ le cycle annuel des saisons en expo-

études religieuses du début du XXe siècle. On fait référence particulièrement à M.P. NiLsson, Primitive Religion, Lund, 1919; id., Primitive Time Reckoning, Lund, 1920.

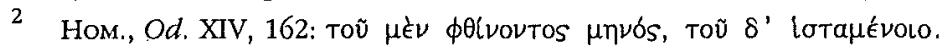

3 M.P. Nissson, Die Entstehung und religiöse Bedeutung des griechischen Kalenders, Lund, 1918, p. 27-29; thèse reprise par le même auteur, in Primitive Time Reckoning, o.c. (n. 1), p. 233.

4 Voir, par ex, M. GuarduccI, "Note sul calendario cretese", Epigraphica 7 (1945), p. 72-89; R. Fischer, "Astronomy and the Calendar in Hesiod", EMC 21 (1977), p. 58-62 (voir p. 62: "Hesiod's Works and Days as a farmer's almanac, is an excellent exemple of a primitif calendar "); J. SARKADY, "A Problem in the History of the Greek Calendar. The Date of the Origin of the Month's Names", $A C D 21$ (1985), p. 3-17.

5 Cf. F. Solmsen, "The Days of the Works and Days", TAPbA 94 (1963), p. 293-320; W. Marg, Hesiod: Sämtlicbe Gedichte übersetzt und erläutert, Zurich, 1970, p. 383-386. Pour une autre approche, voir M.L. West (éd.), Hesiod. Works and Days, Oxford, 1978, p. 346 : "Scholars sometimes call this section "the Days", abstracting the name from Works and Days, but there is no ground for thinking that it was ever considered a separate poem "; également, R. Hamilton, The Architecture of Hesiodic Poetry, Baltimore/ London, 1989, p. 67-77, et A. LardinoIs, "How the Days fit the Works in Hesiod's Works and Days", AJA 119 (1998), p. 319-336.

6 HÉs., Trav. et Jours, 383-828.

7 bid., 383-694. 
sant en même temps les moments favorables ou contraires à l'exécution des travaux de la terre, de la navigation et du commerce. L'expression proverbiale "souviens-toi de faire chaque chose en son temps ${ }^{8}$ renvoie à une isotopie qui traverse le poème et qui se présente sous plusieurs formules: " ne remettez rien ni au lendemain ni au surlendemain ${ }^{9}$, « ayant souvenance des

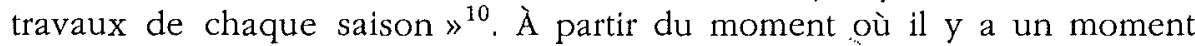
propice pour tout, le poète distingue deux catégories opératoires en vue d'atteindre un résultat analogue : le moment propice et le moment contraire. Le processus de leur identification passe par les indices énonciatifs, comme les impératifs et les adverbes de négation ${ }^{11}$.

Le destinataire du poème ${ }^{12}$, également révélé au moyen d'indices énonciatifs, comme les vocatifs et les pronoms personnels ${ }^{13}$, doit se soumettre à des règles posées par les conditions atmosphériques naturelles et par le mouvement rythmique des planètes, afin de pouvoir gagner la prospérité garantie par l'abondance des produits alimentaires. Il est clair depuis les vers qui précèdent que, face à la prospérité, il n'a pas d'autre choix que celui d'obéir à la volonté de Zeus; une volonté qui est transmise au destinataire par la voix du narrateur, qui de son côté la reçoit par l'intermédiaire des Muses invoquées dans le prélude ${ }^{14}$. Ainsi, de ce fait, le narrateur se présente comme porteur d'un savoir spécifique, conforme à l'aspect didactique de ce type de poésie, destiné à entretenir l'homme par les dons de la terre et leur bonne conservation ainsi que leur échange. La parole par conséquent fonctionne dans un cadre protreptique, dans lequel il est clair que la notion d'un devoir faire qui sort d'une auctoritas est a priori fixé. Dans le cadre du caractère

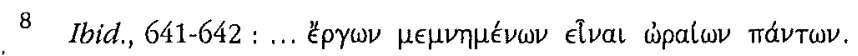

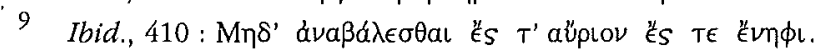

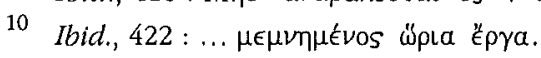

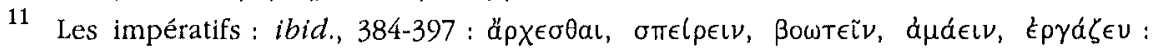
« commencez », « sème », « laboure », « moissonne », « travaille »; les adverbes de néga-

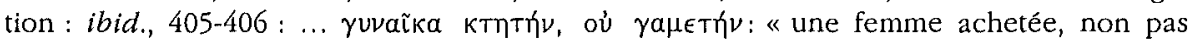
épousée »; ibid., $410: \mu \eta$ : «ne » etc.

12 Voir, à ce propos, P. Pucci, "Auteur et destinataires dans les Travaux d'Hésiode", in F. Braise et al. (éds), Le métier du mythe. Lectures d'Hésiode, Lille, 1996 (Cabiers de Philologie, 16), p. 191-212.

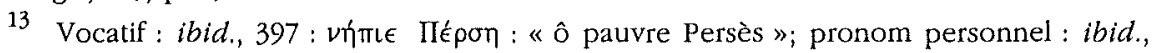
396 : Toı : «à toi "; verbes à la deuxième personne : ibid., $396: \hat{\eta} \lambda \theta \in S^{*}$ " tu es venu ».

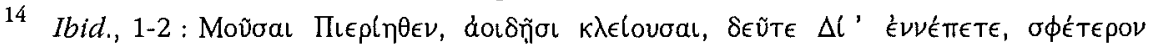

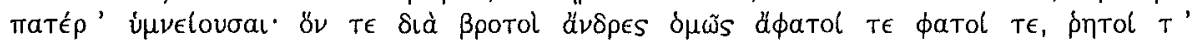

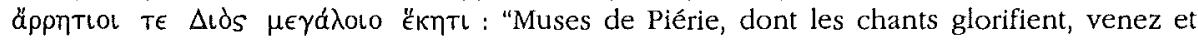
dites Zeus, 'célébrez votre père, par qui tous les mortels sont obscurs ou illustres, connus ou inconnus, au gré de Zeus puissant »; sur le rôle des Muses dans la poésie hésiodique, voir G. Arrighetri, "Hésiode et les Muses : le don de la vérité et la conquête de la parole", in Blaise, o.c. (n. 12), p. 53-70; sur le prélude, voir C. Calame, "Le proème des Travaux d'Hésiode, prélude à une poésie d'action", ibid., p. 169-190. 
didactique de ce type de poésie ${ }^{15}$, les divinités (Zeus, Poséidon, Déméter) ordonnent le rythme de la vie. Leurs champs d'action se manifestent de façon parallèle, sous la forme d'une isotopie qui traverse l'ensemble du poème. Tout d'abord la volonté de Zeus règle le rythme des saisons. À côté de lui, Déméter règle le rythme de la culture céréalière. Poséidon règle le rythme de la navigation parallèlement aux « travaux d'Aphrodite d'or » et aux « dons de Dionysos riche en joies ${ }^{16}$.

Dans les vers 695-764 qui suivent, le narrateur - toujours dans sa fonction protreptique - se dirige vers un plan éthique lui permettant de mettre de nouveau en scène des conseils qui indiquent au destinataire le chemin «véridique » pour gagner sa vie et sa prospérité. Pour une deuxième fois, le destin ( $\beta$ los) de l'homme n'est pas soumis à des circonstances imprévues, mais il dépend toujours de sa soumission ou non à la volonté divine, de son respect ou non de la justice $(\delta(\mathrm{k} \eta)$, ainsi que de ses propres choix pour le moment propice de son mariage, l'équipement de sa maison, les soins de ses animaux, etc. Cette fois-ci, l'ordre du « calendrier» poétique se met en relation avec les étapes de la vie humaine ${ }^{17}$. Il se manifeste par l'utilisation répétitive de mots et d'expressions comme « année » (દ̈ToS), « saison » /

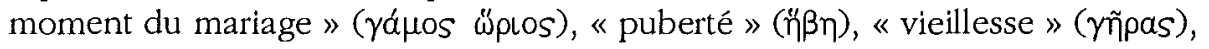

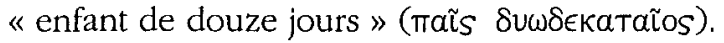

15 M. Heath, "Hesiod's Didactic Poetry", CQ 35 (1985), p. 245-263.

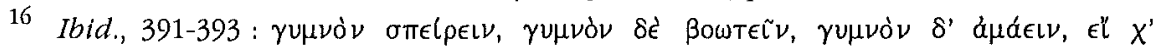

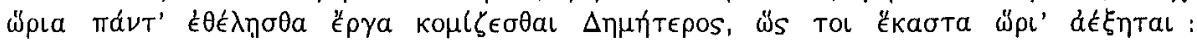
"Sème nu, laboure nu, moissonne nu, si tu veux achever en leurs temps tous les travaux de Déméter, afin que, pour toi, chacun de ses fruits croisse aussi en son temps... »; ibid.,

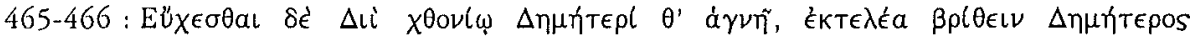

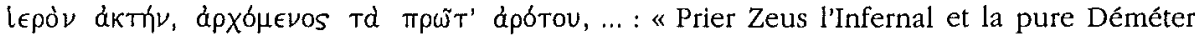
de rendre lourd en sa maturité le blé sacré de Déméter, au moment même où,

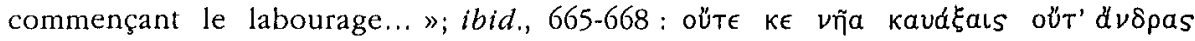

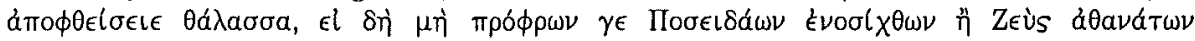

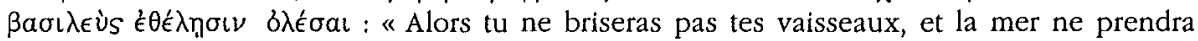
pas tes équipages - à moins que Poséidon qui ébranle la terre, ou Zeus, roi des

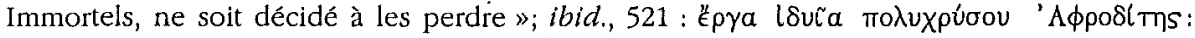

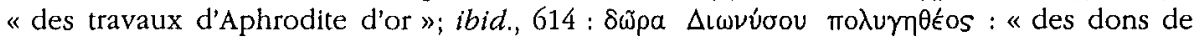
Dionysos riche en joies ".

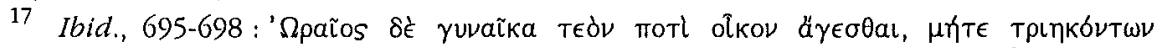

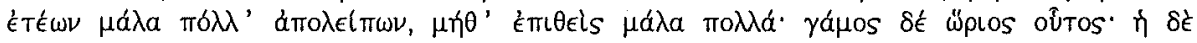

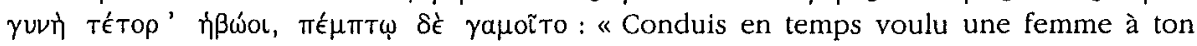
foyer. Pour cela ne devance ni ne dépasse de beaucoup la trentaine. Voilà la vraie saison du mariage. Pour la femme, qu'elle reste quatre années pubère et se marie la

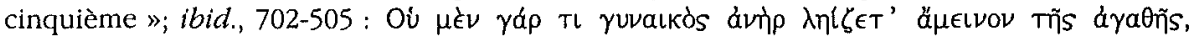

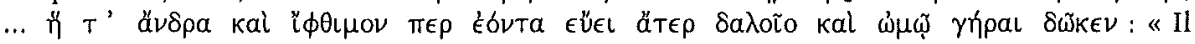
n'est pas pour l'homme de meilleure aubaine qu'une bonne épouse, ..., qui, si vigoureux que soit son mari, le consume sans torche et le livre à une vieillesse prématurée "; ibid.,

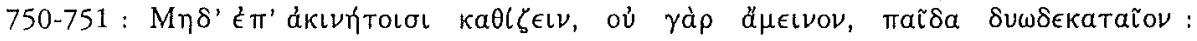
«N'assieds pas non plus - ce n'est pas bien - un enfant de douze jours sur des objets sacrés». 
Dans les vers $764-828$ qui suivent, le poète en suivant toujours un schéma de développement dynamique présente une apparence de " calendrier civil », façonné à des fins poétiques. Les moments favorables ou contraires de la première séquence (cycle des saisons) destinée à la bonne production agricole ${ }^{18}$, sont remplacés sur le plan narratif par des jours spécifiques, favorables ou contraires à l'exécution de diverses activités de la vie quotidienne, dans l'attente d'une prospérité analogue : " Heureux et fortuné celui qui, sachant tout ce qui concerne les jours, fait sa besogne sans offenser les Immortels, consultant les avis célestes et évitant toute faute $»{ }^{19}$ Au niveau de la thématique choisie, on observe une reprise des thèmes des deux premières séquences, classés maintenant non selon des périodes et des saisons propices ou contraires, mais selon des jours propices ou contraires: « Mais méfiez-vous du treizième jour du début du mois pour vous mettre aux semailles. Il est bon en revanche pour planter ${ }^{20}$. La présence divine est une fois encore clairement associée, dans la temporalité narrative, à la chaîne de la production alimentaire, et elle impose ses contraintes à l'état de prospérité des affaires humaines ${ }^{21}$. Pour revenir au système de calcul des jours, il s'agit d'un système qui comporte des mois et des jours qui se répètent selon plusieurs séquences. Les formules de calcul sont multiples et correspondent à des systèmes parallèles en fonction dans les cités grecques ${ }^{22}$ : en trois décades $^{23}$, suivant les phases lunaires ${ }^{24}$, etc. La référence, aux vers 770-771, à la naissance d'Apollon et la qualification du septième jour du mois comme

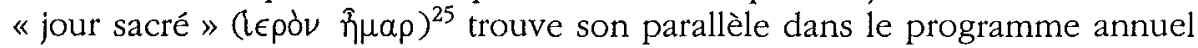

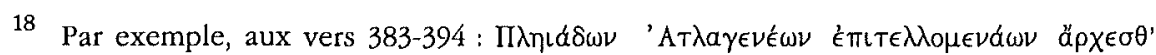

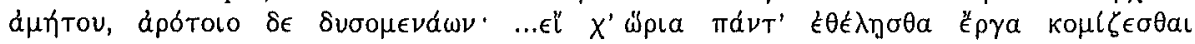

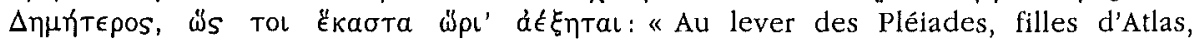
commencez la moisson, les semailles à leur coucher; ... și tu veux achever en leur temps tous les travaux de Déméter, afin que, pour toi, chacun de ses fruits croisse aussi en son temps».

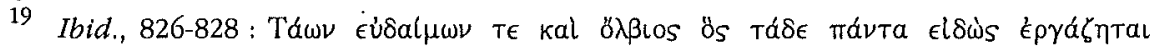

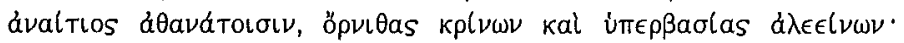

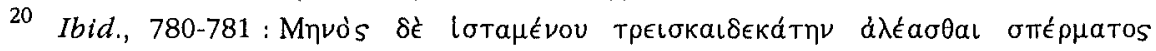

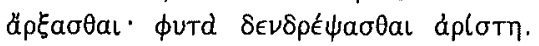

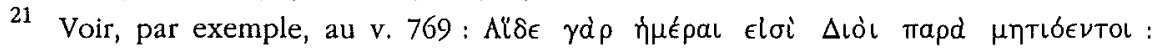
"Voici en effet les jours de Zeus très sage ".

22 WEST, o.c. (n. 5), p. 346.

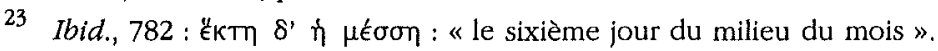

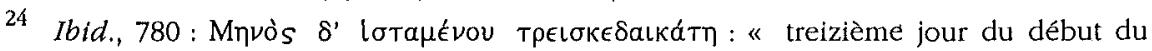

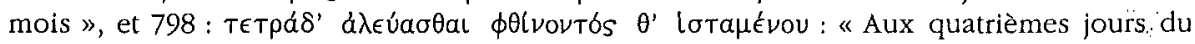
début et de la fin du mois ».

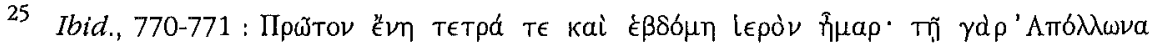

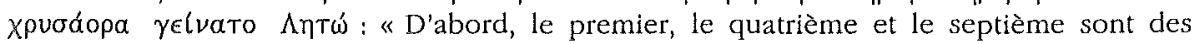
jours sacrés; le septième, Létô enfanta Apollon à l'épée d'or ». 
des cultes de diverses cités ${ }^{26}$. Une telle référence textuelle à la réalité civique s'inscrit aussi dans le développement dynamique de thèmes spécifiques qui caractérisent le poème. On peut aussi distinguer la mention faite aux vers précédents au mois de Lénaiôn ${ }^{27}$, qui est aussi un mois qu'on retrouve dans plusieurs cités ${ }^{28}$. La reproduction circulaire de ces dates dans la dernière partie du poème suit le devéloppement dynamique de la temporalité narrative. Le narrateur commence par le début du mois, ensuite il arrive vers la fin pour recommencer avec d'autres conseils concernant le début du mois, etc.

En somme, le même schéma se produit d'abord pour des saisons et ensuite pour des années, des mois, pour arriver finalement jusqu'aux jours. Il se manifeste par l'emploi d'une série de structures annulaires qui créent l'apparence cyclique d'un programme annuel des événements qui se répètent : dans le cas du cycle des saisons grâce au rappel des solstices et des équinoxes ${ }^{29}$; dans le cas du cycle des mois grâce au rappel de certains moments ${ }^{30}$; et dans le cas des jours, au moyen de la répétition des dates destinées à l'exécution de diverses activités, partant du début du mois pour aller vers sa fin, et de nouveau depuis le début; et aussi par accumulations des nombres des jours.

Pour résumer, on peut dire que la logique du système de la mention des jours est en relation avec le programme poétique. Les efforts qui ont été faits pour la déchiffrer en termes de "calendrier civil » semblent ne conduire nulle part, puisque les dates citées sont ordonnées selon plusieurs systèmes de calcul et avec des fonctions différentes ${ }^{31}$. Mais comment la temporalité narrative achève-elle finalement l'articulation de plusieurs plan annuels que l'on vient de voir, aux vers 383-828, sur la partie du poème qui précède le «calendrier » hésiodique?

Prenons le poème dès les premiers vers, ses principaux thèmes sont la justice $(\delta(\kappa \eta)$ et la nécessité pour l'homme de construire son destin ( $\beta$ los) par

26 M.P. Nilsson, Griecbische Feste von religiöser Bedeutung mit Ausscbluss der attiscben, Darmstadt 1957, p. 97-179; L. Deubner, Attische Feste, Darmstadt, 1962, p. 179204; R. PARKER, "Festivals of the Attic Demes", in T. Linders \& G. Nordquist (éds), Gifts to the Gods, Uppsala, 1987 (Boreas 15), p. 137-147; C. CALAME, Thesée et l'imaginaire athénien. Légende et culte en Grèce antique, Lausanne, 1996, p. 289-324; C. TrümpY, "Feste zur Vollmondszeit: Die religiösen Feiern Attikas im Monatslauf und der vorgeschichtliche attische Kultkalender", $Z P E 121$ (1998), p. 109-115; W.K. PritchetT, "Postscript: The Athenian Calendars", ZPE 128 (1999), p. 79-93.

27. Hés., Trav. et Jours, 504; Voir à ce propos, G. NAGY, Greek Mytbology and Poetics, Ithaca/London, 1990, p. 63 ("Hesiod and the Poetics of Pan-Hellenism").

28 Voir C. TRÜMPY, Untersucbungen zu den altgriecbiscben Monatsnamen und Monatsfolgen, Heidelberg, 1997, p. 293.

29 Hés., Trav. et jours, 478, 561-562, 564, 663.

30 Références aux mois et moments fixés en nombre des jours : ibid., 504, 557, 564565,663 .

31 Ibid., 782 : «le $6^{\mathrm{e}}$ jour du milieu du mois » (= le $19^{\mathrm{e}}$ jour du mois) et au v. 785 : «le $\sigma^{e}$ jour du début du mois ». 
le travail ( $\left.\epsilon^{\prime} \rho \gamma o v\right)$. Dans les vers 11-41, la justice est associée, au moyen du discours sur les deux Luttes, à la notion du travail; les deux isotopies se trouvent donc dans un équilibre bien proportionné. C'est également à ce moment que les divinités apparaissent conformément à leurs champs d'action : Zeus pour la justice et Déméter pour le travail. Après avoir défini le sujet qu'il va développer dans la suite de son poème, le poète cherche un moyen pour l'orienter dans l'espace et le temps, afin de rendre ses paroles valables dans le présent. À cette fin, il va utiliser une série de récits ( $\lambda o ́ \gamma o$ ).

Dans les vers $42-105$, les récits $(\lambda o ́ \gamma o \mathrm{o})^{32}$ de Prométhée et de Pandore ${ }^{33}$ vont désormais illustrer son propos. Les événements racontés dans ces récits se situent à l'époque des Iapétides. La liaison établie aux premiers vers entre la justice et le travail trouve un écho dans les thèmes des récits racontés. Pourquoi la justice et le travail sont-ils mentionnés dans ces récits l'un à côté de l'autre? Parce que le seul moyen de rester juste, selon le poète, c'est de travailler ${ }^{34}$. Et depuis quand ? Depuis le moment où s'est achevée la période où « la race humaine vivait auparavant sur la terre à l'écart et à l'abri des peines, de la dure fatigue, des maladies douloureuses, qui les apportent le trépas ${ }^{35}$. Ensuite, « les dieux ont caché ce qui fait vivre les hommes; sinon sans effort, tu travaillerais un jour, pour récolter de quoi vivre toute une année sans rien faire ${ }^{36}$. Les dieux, suite à la désobéissance de Prométhée qui forme l'aition du passage à la nouvelle ère, ont choisi ce destin pour les hommes. Mais l'homme n'est pas condamné à mort. Le travail lui apportera la prospérité, et son compagnon sera l'Espoir, dont la présence sera constante d'après l'aition de la justice divine fourni par le récit de Pandore. Les deux logoi fournissent une explication des paroles poétiques du début du poème. Ils «fondent» la véracité de ces paroles en remontant à l'événement fondateur de la nouvelle ère, appartenant au passé légendaire. Cet usage du logos par le poète fournit par conséquent l'apparence du début d'un comput chronologique. C'est à partir d'un moment spécifique appartenant au temps raconté, situé dans le passé, que la vie des hommes a changé. Ainsi, la temporalité narrative, qui s'exprime de façon linéaire, crée un comput chronologique à caractère lui aussi linéaire.

Ensuite, le développement dynamique incluant les thèmes principaux qui traversent le poème se poursuit, aux vers 106-201, avec le récit ( $\lambda 6 \gamma_{0 s}$ ) des

32 On préfère garder ici le mot logos, à la place du terme de " mythe », puisque c'est le terme utilisé par Hésiode pour décrire les récits qu'il va raconter dans la suite de son ouvre.

33 Sur le récit de Pandore, voir P. Judet de la Combe \& A. Lernould, "Sur la Pandore des Travaux. Esquisses", in Blaise, o.c. (n. 12), p. 301-314; J.-P. Vernant, « Les semblances de Pandora », ibid., p. 381-392.

$\begin{array}{ll}34 & \text { Ibid., 11-41. } \\ 35 & \text { Ibid., 90-92. } \\ 36 & \text { Ibid., 42-44. }\end{array}$ 
races $^{37}$. La succession des races dans le temps raconté donne l'impression d'un véritable système chronologique, mais qui n'existe que dans la temporalité narrative du poème et à l'aide de marques énonciatives : des adverbes de temps et des adjectifs ordinaux ${ }^{38}$. La référence à l'ancienne ère se fait, au vers 109 , au moyen de l'adverbe de temps prôtista qui caractérise la première race humaine, contemporaine de la production spontanée du sol et de l'absence de douleur pour les hommes. Il renvoie, au moyen d'une structure annulaire ${ }^{39}$, au récit de Pandore, qui fournit, comme on l'a déjà mentionné, l'aition du passage narratif de l'ancienne à la nouvelle ère.

Dans le récit des races, le temps raconté se situe, à l'aide des temps verbaux, au passé; un passé qui se lie ensuite avec le présent de l'énonciation : le « maintenant » ( $\nu \tilde{\nu} \nu$ ) des vers 176 et $202^{40}$. C'est à partir de ce moment que le temps verbal employé est celui du présent. La liaison du passé au présent, à partir du premier «maintenant » et jusqu'au vers 383 du début de la partie appelée "calendrier", est établie au moyen d'une série de structures annulaires qui nous renvoient de façon répétitive à Zeus et Déméter, aux thèmes de la justice et du travail, au motif de l'abondante production du sol, etc $^{41}$. Car les mêmes thèmes, qu'on observe depuis le début du poème, continuent à être déployés. C'est à partir du vers 202, au moment de la fixation des thèmes principaux au «présent » énonciatif, que la quête du moment propice réapparaît pour se poursuivre jusqu'à la fin du poème. Le déploiement des thèmes principaux prend maintenant, à l'aide de structures annulaires, l'apparence cyclique de plusieurs plans annuels: des travaux de la terre, de la navigation, de l'apiculture, etc. Prenant les choses à l'envers, c'est de cette façon finalement que le passé du temps raconté des récits hésio-

37 J.-P. VERNANT, "Le mythe hésiodique des races. Essai d'analyse structurale", $R H R$ 157 (1960), p. 21-56; L. Couloubaritsis, "Genèse et structure dans le mythe hésiodique des races", in BlAise, o.c., (n. 12), p. 479-518; pour une autre approche, voir M. CrubelliER, "Le mythe comme discours. Le récit des cinq races humaines dans Les Travaux et les Jours", ibid., p. 431-463; également J. Fonterose, "Work, Justice, and Hesiod's Five Ages", CPh 69 (1974), p. 1-24: "The myth is a paradigm, an exemplum of his argument, a synchronic scheme presented as history "; voir dernièrement, R. Gotshalk, Homer and Hesiod, Myth and Pbilosophy, Lanham/New York/Oxford (University Press of America), 2000, p. 211-237.

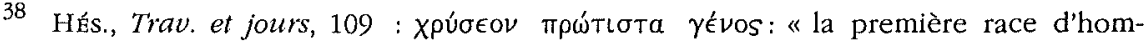

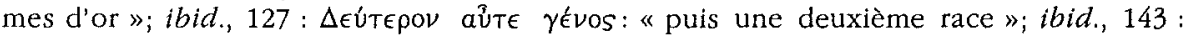

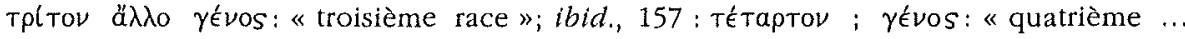

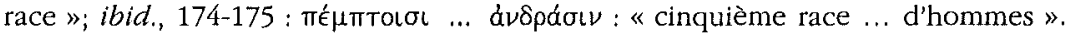

39 Ibid., $90:$ : $\rho l \nu \mu \epsilon^{2} \nu$ : « auparavant».

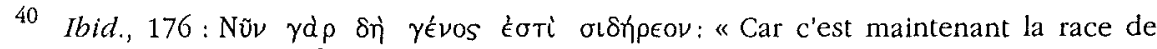

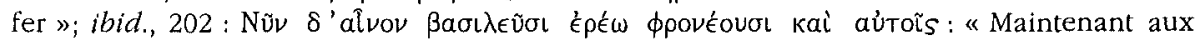
rois, tout sages qu'ils sont, je conterai une histoire ".

41 Pour Zeus : ibid., 180, 245, 267, 273, 333; pour Déméter : ibid., 301; pour la justice : ibid., 213, 226, 249, 256, 270-273; pour le travail: ibid., 399, 306-311. 
diques (logoi de Promethée, de Pandore, des races) ${ }^{42}$ donne une dimension historique aux plans annuels du présent énonciatif ${ }^{43}$. Suite au processus énonciatif choisi (usage des indices énonciatifs, temps verbaux, etc. ${ }^{44}$ ), un plan annuel et sa dimension historique se construisent ${ }^{45}$. Quel est donc le caractère de ce plan annuel construit dans et par le discours? Il est évident que le «calendrier » hésiodique, malgré le fait qu'il se réfère partiellement à la réalité immuable des travaux agricoles n'est pas en fait un traité agricole. Dans le traité agricole postérieur, par exemple, de Palladius ${ }^{46}$, les travaux des champs sont décrits selon le mois de leur exécution et avec le plus grand détail, sans les conseils moraux qui caractérisent la poésie didactique d'Hésiode. Nous pensons que le plan annuel hésiodique et sa mise en perspective historique sont façonnés par le poète afin d'illustrer son propos : la relation entre la justice et le travail. En mettant en scène la liaison étroite entre ces deux notions, il explique en remontant au passé lointain, le début et la raison de cette liaison. Ensuite, il signale le moment propice dans l'année pour l'exécution de diverses activités (plusieurs plans annuels), nécessaire pour garder l'équilibre entre la justice et le travail également au moment présent. En conclusion, et revenant à la question du programme annuel des cultes des cités grecques et donc à la temporalité cultuelle (cyclique), il nous semble que la mention d'un seul nom de mois et la référence à quelques actes cultuels des cités dans la construction narrative du plan annuel chez Hésiode ne nous permettent pas d'utiliser le texte comme terminus ante quem de l'établissement d'un calendrier systématique dans les cités grecques de l'époque « archaïque ». La référence d'Hésiode au programme annuel des cultes des cités fournit simplement une preuve de l'existence d'un tel pro-

42 Pour l'usage du logos dans la littérature grecque, voir C. Calame, "Muthos, lógos et histoire. Usages du passé héroïque dans la rhétorique grecque", L'Homme 147 (1998), p. 127-149; également, C. Calame, Poétique des mythes dans la Grèce antique, Paris, Hachette, 2000, p. 18.

43 On fait ici référence à la performance du poème. Sur la dimension performative de la poésie hésiodique, voir G. NAGY, Poetry as Performance: Homer and Beyond, Cambridge, 1996.

44 Sur le rôle des indices énonciatifs et des temps verbaux dans la description du temps, voir E. Benveniste, Problèmes de linguistique générale, Paris, 1986, II, p. 69-73.

45 Sur la notion du "mythe » et de l'« histoire » dans la Grèce archaïque et classique et l'application moderne de deux termes, voir l'approche de C. Calame (Mythe et Histoire dans l'Antiquité grecque. La création symbolique d'une colonie, Lausanne, 1996); également les études réunies in D. Bouvier \& C. Calame (éds), Pbilosopbes et bistoriens anciens face aux mythes, Lausanne/Paris, 1998 (Études de Lettres, 2); plus spécifiquement, voir D. Bouvier, "Le mythe comme objet historique ou philosophique" (p. 145153).

46 M. Nisard (éd), R.T. Palladius, "De l'Agriculture». Traité d'agronomie antique, Paris, 1999; sur l'agriculture et sa signification dans l'œuvre poétique d'Hésiode, voir S. Nelson, God and the Land: The Metaphysics of Farming in Hesiod and Virgil, Oxford, 1998; et M.S. MarsiLio, Farming and Poetry in Hesiod's Works and Days, Lanham/New York, Oxford (University Press of America), 2000, p. 15-30. 
gramme. Les recherches récentes sur les noms de mois ont montré que ces derniers datent d'avant le vir ${ }^{\mathrm{e}}$ siècle ${ }^{47}$.

On se propose par conséquent de voir dans le récit hésiodique la configuration narrative d'un plan annuel dans la mesure ou la mise en intrigue poétique, comme un philosophe contemporain l'avait montré, compose ensemble des facteurs aussi hétérogènes que « des buts, des moyens, des interactions, des initiatives, des circonstances, des conséquences non voulues issues de l'action humaine ${ }^{48}$. Pour finir, le but de cette étude préliminaire du texte hésiodique n'est pas d'exclure les textes poétiques de recherches qui traitent le programme annuel des cultes des cités grecques; mais au contraire de démontrer que, pour tirer des conclusions sur la construction du temps cultuel d'une période historique, à partir d'une œuvre poétique, il faut se rendre compte de deux temporalités bien distinctes, qui se trouvent en interaction constante à l'intérieur de ce dernier et qui se manifestent de façon parallèle : d'un côté le temps du rituel qui renvoie à une notion cyclique du temps, et de l'autre côté le temps du récit poétique qui se manifeste de façon linéaire ${ }^{49}$.

Université de Lausanne

Sophia Kravaritou EPHE - Paris

La liaison entre la justice et le travail et le rôle du "calendrier"

dans le programme poétique des Travaux et les jours d'Hésiode

\begin{tabular}{|l|l|}
\hline v. 11-41 & Liaison entre la justice et le travail : discours sur les deux Luttes. \\
\hline v. 42-108 & $\begin{array}{l}\text { Aition et événement fondateur de la liaison entre la justice et le travail : } \\
\text { récits de Prométhée et de Pandora. }\end{array}$ \\
\hline v. 109-201 & $\begin{array}{l}\text { Apparence de système chronologique montrant le développement de la } \\
\text { relation entre la justice et le travail, à partir de l'événement fondateur } \\
\text { jusqu'au présent de l'énonciation. }\end{array}$ \\
\hline v. 202-382 & $\begin{array}{l}\text { Discours à propos de la relation entre la justice et le travail au présent } \\
\text { de l'énonciation. Le cboix du moment propice. }\end{array}$ \\
& $\begin{array}{l}\text { Le choix du moment propice : « calendrier hésiodiqué » } \\
\text { a) v. 383-694 } \rightarrow \text { moments favorables ou contraires en rapport avec le } \\
\text { cycle annuel des saisons (travaux de la terre / élevage des animaux / } \\
\text { navigation / commerce) } \\
\text { b) 695-764 } \rightarrow \text { moments favorables ou contraires en rapport avec les } \\
\text { c) v. 765-828 } \rightarrow \text { jours favorables ou contraires à l'exécution des diverses } \\
\text { activités de la vie quotidienne (travaux de la terre / élévage des } \\
\text { animaux / navigation / commerce + conseils étbiques) }\end{array}$ \\
\hline
\end{tabular}

47 Cf. TRÜMPY, o.c. (n. 28).

48 P. Ricoeur, Temps et récit, Paris, 1983, I, p. 11

49 Sur les interactions entre différents régimes temporels dans les textes de la Grèce antique, voir les études réunies par C. DARBo-Peschanski (éd.), Constructions du temps dans le monde grec ancien, Paris, CNRS Éditions, 2000. 